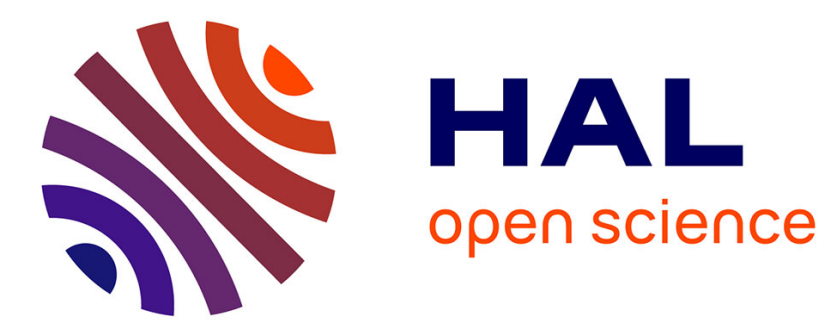

\title{
De nouvelles phonologies? Sur quelques évolutions récentes de la phonologie générative
}

\author{
Denis Costaouec
}

\section{To cite this version:}

Denis Costaouec. De nouvelles phonologies? Sur quelques évolutions récentes de la phonologie générative. La linguistique: revue internationale de linguistique générale, 2002, 38-2, pp.139-158. 10.3917/LING.382.0139 . halshs-00591056

\section{HAL Id: halshs-00591056 \\ https://shs.hal.science/halshs-00591056}

Submitted on 6 May 2011

HAL is a multi-disciplinary open access archive for the deposit and dissemination of scientific research documents, whether they are published or not. The documents may come from teaching and research institutions in France or abroad, or from public or private research centers.
L'archive ouverte pluridisciplinaire HAL, est destinée au dépôt et à la diffusion de documents scientifiques de niveau recherche, publiés ou non, émanant des établissements d'enseignement et de recherche français ou étrangers, des laboratoires publics ou privés. 


\section{2, «DE NOUVELLES PHONOLOGIES ? SUR QUELQUES EVOLUTIONS RÉCENTES DE LA PHONOLOGIE GÉNÉRATIVE», LA LINGUISTIQUE, VOL. 38-2, PARIS, PUF, p. 139-158.}

\section{DOI : 10.3917/LING.382.0139.}

In which way can the functionalists be interested in generative phonology? This paper exposes some changes in the generative thesis - developed, since the 1970's, as a response to the weakness of the Chomsky and Halle's "S.P.E. model". It also tries to define the converging and diverging view points of generative phonology with the functionalist approach.

La phonologie générative s'est transformée depuis une trentaine d'années en réaction au modèle développé par Noam Chomsky et Morris Halle dans leur ouvrage The Sound Pattern of English ${ }^{1}$. Pour ces auteurs, la phonologie était conçue comme un sous-produit de la syntaxe générative, comme un ensemble de règles de réécriture aboutissant à l'expression phonique d'une suite générée par la syntaxe. La critique de ces positions par les générativistes a commencé avant même la publication de The Sound Pattern of English, à l'occasion de différents colloques et de la publication de nombreux articles. Très tôt, la subordination complète de la composante phonologique à la composante syntaxique a posé problème, sans que celle-ci ne soit ouvertement remise en cause jusqu'à nos jours. Les propositions de Chomsky et Halle ont fait l'objet de multiples réarrangements qui visaient à sortir du cadre limité, strictement linéaire et dérivationnel, des «règles de réécriture» et à traiter des domaines qui restaient extérieurs au «modèle S.P.E.» (pour The Sound Pattern of English) comme les phénomènes syllabiques et quantitatifs, les phénomènes prosodiques, rythmiques et accentuels, les phénomènes supra-segmentaux, les phénomènes «morphophonologiques».

Le modèle S.P.E. a été progressivement abandonné au profit de propositions théoriques, qui - si elles ne sont pas toutes compatibles entre elles - ont en commun de viser l'établissement de «représentations des structures phonologiques», conçues comme des configurations, une sorte de géométrie ou d'architecture, qui veulent décrire les principes d'auto-organisation de la composante phonologique (c'est-à-dire, on le verra plus loin, échappant à un modèle où les principes logico-syntaxiques d'une sorte de «langage mental» assureraient la transformation des séquences de morphèmes en représentation phonique puis en séquences sonores). Différents courants se partagent le domaine, comme la phonologie métrique ou la phonologie autosegmentale $^{2}$, chaque branche principale ayant produit à son tour de multiples

${ }^{1}$ CHOMSKY, Noam et Morris HALLE, The Sound Pattern of English, New-York, Harper \& Row, 1968. Publié en français en 1973 sous le titre Principes de phonologie générative, Paris, Éd. du Seuil, 349 p., (traduction par Pierre Encrevé).

2 On peut considérer que ces deux courants ont le plus marqué l'évolution de la phonologie générative de ces dernières décennies. 
variantes $^{3}$. Ces approches appuient de nombreux travaux sur la chaîne sonore (notamment sur les tons, l'accent, l'harmonie vocalique, le découpage syllabique, la liaison...), et étayent les thèses d'un courant dit phonologie cognitive dont il sera question également.

On tentera de présenter ici les préoccupations de ces nouvelles tendances - souvent concurrentes et généralement parcellaires -, les conditions dans lesquelles elles ont émergé et les réflexions qu'elles peuvent inspirer du point de vue de la phonologie structurale et fonctionnelle.

Il a fallu faire des choix de lecture dans une littérature surabondante, s'adapter à la terminologie et résister à l'abattement que peut provoquer ce genre de prose chez le fonctionnaliste le mieux disposé... Une des difficultés de l'exercice étant de simplifier les thèses générativistes pour les besoins de l'exposé, sans les caricaturer, au risque d'être parfois un peu elliptique: pour plus de précisions le lecteur intéressé pourra se reporter aux références bibliographiques fournies. La base d'enquête est donc partielle, guidée par des auteurs tels que Laks, Encrevé, Carvalho ou Angoujard, vers des chercheurs comme Lowenstamm, Selkirk, Kaye, Clements, Goldsmith, Prince ou encore Halle et Vergnaud, qui sont à l'origine des réorientations de ces vingt dernières années mais dont certains travaux restent hors d'atteinte pour un linguiste qui n'a pas décidé d'investir de longs mois dans la compréhension des subtilités de la pensée générativiste.

Dans le cadre d'un tel article, il s'agira surtout de pointer les domaines où la démarche générativiste et la démarche fonctionnelle restent fondamentalement distinctes et ceux où des préoccupations communes peuvent émerger: si l'hypothèse d'une «phonologie universelle» reste vide de sens pour les fonctionnalistes et qu'ils n'assignent pas à la phonologie la tâche de rendre compte de certaines facultés cognitives des locuteurs ${ }^{4}$, en revanche, la nature du phonème, le statut de la syllabe, les mécanismes de l'accentuation, l'explication des phénomènes d'harmonie vocalique, ou encore l'adéquation de la théorie aux faits et sa capacité explicative... sont des problèmes qui préoccupent tout phonologue.

\footnotetext{
${ }^{3}$ Elles constituent ce que les générativistes nomment eux-mêmes les «Nouvelles phonologies»: Morphologie Nonconcaténative, Théorie de la Sous-spécification, Phonologie à Contraintes et Réparations, Phonologie du Charme et du Gouvernement, Phonologie de Dépendance, Phonologie Déclarative, Phonologie de Laboratoire... Pour s'y retrouver un peu dans ce foisonnement, on consultera avec intérêt le numéro 125 de la revue Langages (Paris, Larousse, mars 1997, 125 p.), dirigé par Bernard LAKS et intitulé précisément «Nouvelles phonologies»; le numéro 17 des Recherches linguistiques de Vincennes, publié en 1988 sous la direction de Pierre ENCREVÉ et intitulé également «Nouvelles phonologies»; ainsi que le livre de John GOLDSMITH, The Handbook of Phonological Theory, Cambridge, Mass., Blackwell, 1995.

4 Si une certaine conception de «l'esprit humain» émerge des thèses fonctionnalistes, c'est sans doute celle d'un objet fluent où tout est constamment en cours de transformation, et rien dans la démarche ne privilégie l'hypothèse d'un «codage unique» de l'information. Ce point de vue, paradoxalement, est plus proche des enseignements récents des sciences cognitives que le modèle cybernéticien qui domine, ou a longtemps dominé, certaines tendances générativistes. Et au-delà, les thèses fonctionnalistes pencheraient sans doute du côté de la «cognition située», dont le centre de préoccupations est plutôt l'homme en société que le sujet au singulier, psychique ou physiologique.
} 
L'époque est révolue où les générativistes se cantonnaient, à la suite de leurs chefs de file, au travail sur l'anglais et quelques autres langues occidentales de grande diffusion. S'appuyant sur les descriptions existantes (œuvres de structuralistes bien souvent) ou sur des travaux originaux, ils ont frotté leurs thèses à diverses langues et ont été contraints d'introduire plus de réalisme dans leur raisonnement (par exemple en reconnaissant que la substance phonique peut être autre chose qu'une simple traduction in fine d'une réalité systématique immatérielle, la seule qui serait proprement linguistique... $)^{5}$. Il ne s'agit pas d'un effet mécanique de la confrontation à la diversité des langues - jusqu'à aujourd'hui les faits empiriques n'ont d'intérêt pour bien des générativistes qu'en tant qu'ils permettent d'éprouver leurs hypothèses théoriques - ou d'une sorte de découverte tardive des vertus du structuralisme (bien qu'on puisse lire sous la plume d'auteurs comme John Goldsmith des références explicites aux travaux de structuralistes bloomfieldiens comme Hockett) ${ }^{6}$, mais plutôt d'une évolution de la théorie du fait de ses contradictions internes, de l'évolution de disciplines connexes comme les sciences cognitives et, plus profondément sans doute, d'une manifestation de l'opposition permanente entre idéalisme et matérialisme dans la réflexion scientifique. Si l'on se garde de voir tout l'idéalisme du côté de la phonologie générative et tout le matérialisme chez les structuralistes, il s'agit cependant de tendances («profondes», c'est le cas de le dire) qui agissent au sein des théories.

\section{Le «modèle S.P.E.»}

Selon le «modèle standard S.P.E.», parmi les composants essentiels d'une grammaire générative, le composant phonologique est conçu comme agissant sur la suite de «morphèmes» produite par les composants syntaxique et sémantique qu'il traduit en un métalangage (représentation) phonétique rendant compte de la prononciation réelle (séquence sonore ou forme sonore).

Dès l'origine, Chomsky et Halle refusent une structure purement phonologique (au sens traditionnel de phonologie) pour des raisons «d'économie» (à comprendre comme «économie formelle», simplicité des modèles): pour construire une représentation phonétique d'une phrase à partir de sa représentation comme suite structurée de morphèmes il est inutile de passer par l'intermédiaire d'une représentation phonologique retenant les traits pertinents et eux seuls; des lois plus simples et plus générales peuvent être formulées en considérant que la suite des sons constituant physiquement le mot découle directement de son organisation en morphèmes. La composante phonologique est conçue comme intégrant de manière indifférenciée ce que d'autres courants distinguent soigneusement: la phonétique, la phonologie et la morphologie. C'est pourquoi, pour rendre compte des opérations qui établissent la structure phonologique des «morphèmes» il a été question un temps de «représentations morphophonémiques» (niveau représentationnel mental, par

\footnotetext{
5 Voir ce qu'en disent Bernard LAKS et Marc PLENAT dans l'introduction à l'ouvrage collectif, De natura sonorum. Essais de phonologie, Saint-Denis, Presses Universitaires de Vincennes, 1993, p. 7 notamment.

${ }^{6}$ Notamment dans John GOLDSMITH, Autosegmental and Metrical Phonology, Cambridge, Mass., Blackwell.
} 
opposition au niveau matériel de la phonétique); ce terme a ensuite été rejeté par Chomsky et Halle (Principes, p. 38) car il implique la reconnaissance du niveau du phonème dont ils pensaient avoir montré l'inexistence ${ }^{7}$.

Cette conception de la production sonore - qui rejette le phonème, la notion de système phonologique et ignore syllabe et phénomènes prosodiques -, s'appuie initialement sur un modèle linéaire de règles dérivationnelles dont l'horizon unique est la chaîne parlée. Une règle de transformation ou de réécriture agit, par convention, sur la séquence modifiée par la règle qui la précède, et ainsi de suite. Par exemple, Chomsky explique dans Le langage et la pensée 8 que face à ang. right, prononcé [rayt], il faut postuler une forme sous-jacente /rixt/ qui va être transformée une première fois en [rīt] - en application de la règle selon laquelle «voyelle + vélaire donne voyelle tendue», [rīt] se transformant en [rayt] en fonction d'une autre règle selon laquelle $« / 1 /$ devient [ay]»...

Le «segment» sur lequel agissent les règles est défini, sur le modèle des mathématiques, comme un ensemble ordonné d'éléments qui sont compris dans un intervalle. Il s'agit d'un complexe de traits distinctifs occupant une place donnée dans la chaîne sonore. Les traits distinctifs sont notés à l'aide de traits binaires (pour l'essentiel empruntés aux travaux de Jakobson, Fant et Halle) qui se veulent à la fois un outil formel et un alphabet phonétique universel ${ }^{9}$. Les traits distinctifs retenus par Jakobson ont été complétés par Chomsky et Halle de quelques nouveaux traits, définis $\mathrm{du}$ point de vue articulatoire; au contraire d'autres traits, dont la définition est clairement acoustique, tels compact / non compact, diffus / non diffus, etc., ont été rejetés pour la définition des voyelles et strictement réservés à la présentation du système consonantique. Au fil du temps la description des «segments» va intégrer, outre les traits phoniques, une série de «diacritiques» concernant le caractère «syllabique» de telle unité, l'accent ou encore des règles d'exception; le tout mis sur le même plan...

Employés par Chomsky et Halle, les traits binaires ne visent pas à décrire la matérialité sonore de la langue, considérée comme relativement accessoire. Les segments ainsi décrits sont des objets théoriques, de caractère abstrait, qui entrent dans des opérations formelles. Dans ce cadre, la notation du son sous la forme d'un symbole phonétique, concession ultime à la substance, n'intervient que pour signaler la réalisation matérielle d'une représentation phonologique sous-jacente. On est au cœur d'une phonologie symboliste qui s'efforce de caractériser les propriétés sonores en les distinguant de leur matérialité. De manière plus caractéristique encore, un trait

\footnotetext{
${ }^{7}$ Sur la filiation entre les thèses de Chomsky et celles de Bloomfield, via Harris (notamment sur les notions de règles phonologiques et sur le terme morphophonologie) et sur le déni de cet héritage structuraliste américain par les générativistes convaincus des années 1970-80, voir l'article de Pierre Encrevé, L'ancien et le nouveau : quelques remarques sur la phonologie et son histoire, dans Bernard LAKS (éd.), Langages n 125, p. 100-123.

${ }^{8}$ CHOMSKY Noam, 1990 (1e édition 1970), Le langage et la pensée, Paris, Payot, 144 p.

9 On ne reviendra pas ici sur les critiques qui ont été faites au «binarisme» par Leonard Bloomfield, André Martinet et quantité d'autres auteurs. Au-delà des diverses objections qui peuvent être faites au nom du réalisme linguistique, la proposition de Jakobson et de ses continuateurs repose fondamentalement sur un choix épistémologique, qui conçoit la communication elle-même comme une série de choix binaires (voir notamment l'article de Morris Halle, In defense of the number Two, dans E. Pulgram (éd.), Studies presented to Joshua Whatmough, La Haye, Mouton, 1957, p. 65-72).
} 
phonétique n'est jamais envisagé dans le modèle S.P.E. qu'en tant qu'il est adéquat à la description en termes de règles des processus phonologiques étudiés; fondamentalement son adéquation empirique reste secondaire.

Il y aurait quelque intérêt théorique à se pencher sur le traitement réservé aux traits binaires dans l'évolution de la phonologie générative. On peut évoquer ici quelques points d'achoppement parmi d'autres.

1) Un arbitraire fondamental préside au choix des traits caractérisant un segment et rend toute spécification possible en fonction de la valeur par défaut choisie par le descripteur: par exemple, si l'on considère par hypothèse ou par convention que /i/ est une voyelle «neutre», /e/ sera [- haut], /a/ sera [+ bas], /o/ sera [- haut, + arrière], etc.

2) Dans le modèle S.P.E., le système binaire a en fait trois positions, la troisième étant la position nulle [ ] qui caractérise les segments «non concernés par le trait». Les critiques faites à cette formalisation «ternaire» ont conduit à l'abandon de la marque nulle et, jusqu'aux années 1980, à une «sur-spécification» des matrices phonologiques qui interdisait l'identification «naturelle» de la marque distinctive du segment ainsi défini (cette surabondance de + et de - ne faisant que renforcer le caractère abstrait de la définition du segment).

3) Autre problème, abordé dès 1968 dans The Sound Pattern of English, les deux valeurs des traits binaires n'ont pas le même statut, les mêmes capacités à se propager ou à apparaître dans un contexte donné et ne peuvent donc être considérées comme réellement symétriques...

4) Nouvelle difficulté, les traits binaires se sont montrés «trop riches» pour la théorisation proposée par la phonologie générative: si une marque unique définit assez facilement les oppositions au sein d'un système phonologique, les choses se compliquent sérieusement avec une approche binaire qui nécessite un arsenal pesant de règles de transformations pour rendre compte de la distinction entre des segments proches (voir les contorsions du modèle S.P.E. pour établir la différence entre «U« et «u» par exemple)...

\section{Faiblesses internes d'une phonologie idéaliste et symboliste}

La conception de la phonologie véhiculée par le modèle S.P.E. nie toute autonomie aux faits phoniques, ce que les thèses structuralistes admettent implicitement ou explicitement, et limite le composant phonologique à un simple rôle d'interprétation. Les règles phonologiques sont conçues comme cycliques quand elles visent à prédire des formes phonétiques selon des principes réguliers (c'est le cas quand Chomsky traite du problème des voyelles réalisées [ə] en syllabe atones de l'anglais par exemple ${ }^{10}$ ), mais quand il s'agit de rendre compte de la complexité des énoncés réellement attestés, elles s'accumulent en un large inventaire de réajustements. Ces réajustements sont du même type que les «transformations» affectant le composant syntaxique, qui au fil de l'évolution de la théorie initiale, vont peu à peu occuper toute la place et rendre le modèle inutilisable, à tel point que Chomsky lui-même en viendra,

${ }^{10}$ CHOMSKY, Le langage et la pensée, p. 67-69. 
en 1981, à abandonner le système des règles au profit d'un autre modèle dit «principes et paramètres» ${ }^{11}$.

La règle générative n'est au mieux qu'une présentation synoptique des phénomènes car, fondamentalement, c'est la règle qui découle des régularités observables dans les langues et non l'inverse ${ }^{12}$. De plus, une véritable contradiction mine le projet dès le départ puisqu'il s'agit, par principe, d'atteindre la règle la plus «simple» pouvant décrire un phénomène, règle réputée la plus adéquate, alors que dans la pratique la complexité des faits amène les auteurs à multiplier les emboîtements de règles, les exceptions ou - mieux - les «règles d'effacement» qui annulent l'effet d'autres règles dont on a postulé l'action auparavant...

On trouvera ci-dessous, à titre d'exemple, la formalisation de la règle numérotée (137), présentée dans les Principes de phonologie générative et censée expliquer les conditions d'élision du /i/ dans le suffixe /-isk-/ du russe au sein de séquences comme /sibírskəy/ «sibérien», /rímskəy/ «roumain», etc. Cette règle incluant l'emploi d'une autre règle (134) et d'une condition d'exception...

$$
\left[\begin{array}{l}
+ \text { voc } \\
- \text { cons } \\
+ \text { haut } \\
- \text { arr } \\
- \text { tendu }
\end{array}\right] / \rightarrow \text { règle (134)] }\left[\begin{array}{l}
+ \text { cons } \\
- \text { ant } \\
-\mathrm{D}
\end{array}\right]+- \text { sk }+
$$

qui s'interprète ainsi: /i/ (dans le segment -isk-), ici défini comme [+ vocalique, consonantique, +haut, -arrondi, - tendu] se transforme (ou se réécrit) $(\rightarrow$ ) en application de la [règle (134)] devant (/) [+ consonantique, -antérieur]; [sauf exception: diacritique - D indiquant qu'il ne s'agit pas d'une exception à la règle (137)], et est supprimé (- sk). La règle (134) décrivant elle-même la suppression de /i/ devant syllabe à voyelle tendue... et ainsi de suite.

Outre la complexité des formules et leurs bizarreries qui ont beaucoup occupé les commentateurs, il faut constater que loin d'expliquer - ce qui était l'ambition affichée ce type de présentation des faits restait strictement descriptif, remplaçant - comme le

11 Voir notamment Noam CHOMSKY, Principles and Parameters in Syntactic Theory, dans N. HORNSTEIN et D. LIGHTFOOT (éd.), Explanations in Linguistics, the logical Problem of Language Acquisition, Londres, Longman, 1981, p. 32-75

12 La place manque ici pour développer, mais dans une large mesure les «principes «qui ont succédé aux «règles «fonctionnent selon la même logique. Soit une langue comme le berbère où une séquence */bVf/ (où $\mathrm{V}$ représente n'importe quelle voyelle) est impossible (= non attestée). Pour expliquer ce phénomène on fait appel au Principe du Contour Obligatoire (PCO) selon lequel «deux occurrences adjacentes du même trait sur la même ligne sont exclues»; la démonstration consiste alors à mettre en évidence que puisque /b/ et/f/ partagent le trait [labial], alors qu'en application d'un autre principe relatif aux langues à «morphologie gabaritique «le descripteur les a placés côte à côte sur une même ligne autosegmentale, la séquence */bVf/ est impossible car violant le PCO... CQFD. Le lecteur craignant d'avoir affaire à un résumé tendancieux se reportera avec intérêt à l'article de George CLEMENTS, Lieu d'articulation des consonnes et des voyelles: une théorie unifiée, dans Bernard LAKS et Annie RIALLAND (dir.), Architecture des représentations phonologiques, Paris, CNRS Editions, 1993, pages 117 et 118. 
notait Hagège - un paragraphe de la grammaire traditionnelle par une symbolisation mathématisante, manipulant des objets sans réalité substantielle ${ }^{13}$.

Au bout du compte, quelle que soit l'élégance de l'enchaînement de règles proposé, le résultat ne peut être que trivial: pour rendre compte de la «structure de surface», la seule observable, le phonologue générativiste postule une représentation sous-jacente et sur cette base construit un édifice qui aboutit avec plus ou moins de facilité... à la représentation de surface. Cela nous renseigne sur ses qualités de logicien mais ne peut en aucun cas valider l'hypothèse de la représentation sous-jacente. De plus - l'objection a été faite maintes fois -, le processus n'est pas réversible et jamais une représentation sous-jacente n'a pu être «découverte» à partir d'un quelconque jeu de règles appliquées à un énoncé réel.

Dans une large mesure, on peut dire que cette notion de règle a vécu. Les phonologues générativistes les plus conséquents ont perçu à quel point le «langage de la règle» posait problème du point de vue explicatif, du point de vue de l'idéalisme symboliste qui le fondait, mais aussi par la confusion qu'il maintenait entre faits de synchronie et faits diachroniques, ou encore par la négation du caractère social et conventionnel des langues qu'il impliquait... Et tout en restant, en général, dans le cadre étroit de l'explication dans la chaîne sonore, les modèles ont évolué pour donner la priorité aux relations qu'entretiennent entre elles, même à distance, les entités phonologiques. Les propositions des courants dits phonologie autosegmentale et phonologie métrique notamment, ont été élaborées comme réponse aux impasses de la phonologie purement symboliste et algorithmique du modèle S.P.E., dès lors qu'il s'agissait de lier phonologie et phonétique, dès lors qu'il s'agissait de rendre compte de phénomènes réels constatés dans des langues variées, y compris d'aspects aussi courants que l'accentuation ou les tons.

\section{Vers la phonologie plurilinéaire}

Les différentes étapes de l'abandon des thèses chomskyennes initiales, par Chomsky lui-même et ses continuateurs, donnent la mesure de l'effort qu'il a fallu fournir pour sortir de l'impasse du modèle S.P.E.

Il est assez frappant de constater que les principales critiques formulées par les générativistes à l'encontre du modèle de Chomsky, dans les années 1970-1980, concernent son aspect «linéaire», ce qui n'apparaissait pas aux yeux d'autres courants comme son plus grave défaut; les critiques concernant la soumission de la phonologie à la syntaxe s'expriment également mais de manière assez timide.

On comprendra sans doute un peu mieux la volonté des générativistes d'aboutir à des représentations structurées (non linéaires) des processus phonologiques si l'on se rappelle certaines avancées, dans les années 1970 et 1980, des jeunes «sciences cognitives», fortement influencées par la recherche en informatique. Ces évolutions ont eu un fort retentissement sur les travaux menés dans la ligne chomskyenne du fait des liens étroits qui unissent traditionnellement la linguistique générative aux recherches sur les langages formels et au cognitivisme. Dans les années 1970, les travaux sur la traduction automatique et l'intelligence artificielle remettent en cause les propositions

13 HAGEGE, Claude, 1976, La grammaire générative : réflexions critiques, Paris, P.U.F., coll. «Le linguiste», $244 \mathrm{p}$. 
de la cybernétique des années cinquante: l'information n'est plus conçue comme une accumulation de chaînes de caractères binaires, mais comme un ensemble structuré (en «arbres») d'opérations abstraites; il s'agit d'une part d'établir des «types de données» et d'autre part de hiérarchiser les procédures de manipulation de ces données. En phonologie générative, ces principes vont conduire à l'abandon des listes de traits non structurées du modèle S.P.E. au profit de regroupements de données ayant des caractéristiques communes, et soumises à des traitements particuliers. Une intense réflexion est menée pour définir une hiérarchie des éléments manipulés, qu'il s'agisse de préciser les rapports entre faits segmentaux et faits suprasegmentaux (accentuation par exemple), de prendre en compte les problèmes de quantité ou encore de définir la structure interne des segments sonores.

Dans les années 1980 un pas supplémentaire est franchi dans le domaine des sciences cognitives avec l'adoption des modèles «neuronaux», inspirés du fonctionnement des réseaux de processeurs interconnectés (synapses): ils imposent une conception plus flexible du fonctionnement mental, dépendant surtout de l'architecture et de la complexité du réseau de connexions synaptiques. Ces nouvelles conceptions poussent au rejet de la notion de «langage mental» organisant le traitement de l'information sur une base logico-syntaxique ${ }^{14}$. En phonologie générative, ces conceptions vont renforcer les travaux qui défendent le principe d'une autoorganisation de la composante phonologique (contre sa soumission à la syntaxe) et rejettent (timidement d'abord puis avec plus de fermeté) le postulat des deux niveaux de représentation phonologique - le niveau abstrait et celui de la réalisation effective.

\section{Autosegments et organisation hiérarchique des faits}

Les travaux de Liberman et Prince ${ }^{15}$, puis ceux de Selkirk et de Nestor et Vogel, introduisent dans l'analyse de la chaîne parlée une notion de suprasegmentalité qui désigne une organisation hiérarchique de la chaîne sonore. La proposition concerne à la fois la nature des segments phonologiques et le rapport entre phonologie et syntaxe; ainsi en 1978 Elizabeth Selkirk ${ }^{16}$ considère que la structure prosodique est une organisation suprasegmentale et elle postule que les constituants (phoniques) ont une autonomie relative par rapport à la structure syntaxique. Pour ne prendre qu'un exemple, dans le cadre du modèle S.P.E. l'accent est analysé comme une propriété intrinsèque des segments, au même titre que les autres traits binaires. Pour Selkirk, le contraste accentuel ne résulte pas des propriétés des segments mais de propriétés relationnelles qui opposent les «nœuds» d'une arborescence qui sont affectés d'un

\footnotetext{
14 Pour une introduction générale à ces problèmes, voir entre autres Jean-Gabriel GANASCIA, Les sciences cognitives, Paris, Flammarion, 1996, 125 p. ou G. VIGNAUX, Les sciences cognitives: une introduction, Paris, La Découverte, 1992. Voir également B. LAKS, Langages n 125, p. 9; et B. LAKS, Le connexionnisme, les représentations et la question des niveaux, Cahiers de praxématique $\mathrm{n}^{\circ}$ 31, 1998, p. 149-176.

15 LIBERMAN, M. et A. PRINCE, On Stress and Linguistic Rhythm, Linguistic Inquiry 8, 1977, p. 249-336.

16 SELKIRK, Elizabeth, The French Foot: on the statute of «mute «e, Studies in French Lingusitics, 1
} 
facteur «fort» ou «faible»: le modèle accentuel résulte de la composition de tels contrastes pouvant intervenir à différents niveaux hiérarchiques.

Une des idées importantes avancées par des auteurs comme McCarthy et Prince ${ }^{17}$ est que les représentations phonologiques sont dominées par une structure prosodique hiérarchisée. Différentes hypothèses sont formulées pour rendre compte du caractère hiérarchisé et arborescent des constituants de la chaîne sonore (cette notion d'arborescence s'inspirant en partie des arborescences syntaxiques de la grammaire générative - bien que certains de ses promoteurs s'en défendent). La phonologie métrique propose par exemple une représentation fondée sur le mot phonologique (noté $\omega$ ), le mot prosodique (noté $\lambda$ ), le pied (noté $\Sigma$ ) et la syllabe (notée $\sigma$ ), chaque niveau étant le lieu de certaines propriétés. Ces configurations seront par la suite critiquées pour leur opacité et leur abstraction excessives, y compris par Prince luimême qui propose un autre modèle, fondé sur une «grille rythmique» ${ }^{18}$ qui doit suffire à désigner les segments «proéminents» (segments accentués, signalés dans le schéma par les deux X superposés).

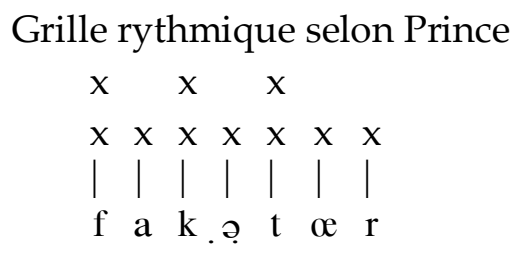

Pour rendre compte des phénomènes de quantité (voyelles longues, consonnes géminées...), différentes voies sont explorées, qui remettent parfois à l'honneur des notions fort anciennes, comme le fait Hayes ${ }^{19}$ en reprenant le découpage en mores, rebaptisées «unités de poids».

\begin{tabular}{|c|c|c|c|c|}
\hline \multicolumn{5}{|r|}{ Représentations moraïques } \\
\hline 1 & 2 & 3 & 4 & 1: voyelle brève \\
\hline$\mu$ & $\mu \mu$ & & $\mu$ & 2: voyelle longue (2 mores) \\
\hline T. & |j & & | & 3: consonne brève (pas de poids moraïque) \\
\hline $\mathrm{v}$ & $\mathrm{v}:$ & c & $\mathrm{CC}$ & 4: consonne géminée \\
\hline
\end{tabular}

Dans le même temps, des travaux sur la syllabe mettent au centre de la réflexion la structure rythmique (grille rythmique de Prince) et la structure prosodique (voir l'historique qu'en fait Angoujard). ${ }^{20}$

\footnotetext{
17 McCARTHY, John \& Alan PRINCE, Prosodic Morphology, Ms., University of Massachusetts, Amherst, and Brandeis University, Waltham, Mass.

18 PRINCE, Alan S., 1983, Relating to the grid, Linguistic Inquiry, 14 (1), p. 19-100.

19 HAYES, Bruce, Compensatory lenghtening in moraic phonology, Linguistic Inquiry, 20 (2), 1989, p. 253-306.

20 ANGOUJARD, Jean-Pierre, 1997, Théorie de la syllabe. Rythme et qualité, Paris, CNRS Editions, coll. Sciences du langage, $224 \mathrm{p}$.
} 
Ces conceptions, largement développées au cours des années 1980 et 1990, vont pour une part dans le même sens (sortir de la pauvreté du modèle S.P.E.) mais s'opposent souvent sur les questions de la proximité à la réalité phonique et de la simplicité. Nouveaux modèles et contre projets, bien souvent incompatibles, se répondent à travers des centaines $\mathrm{d}$ 'articles, témoignant du caractère inabouti mais collectif des réflexions entamées. Ces débats vont déboucher sur quelques propositions importantes pour la phonologie générative: la définition des autosegments (notion proposée par Goldsmith dès 1976) ${ }^{21}$ et une conception plurilinéaire des représentations phonologiques. ${ }^{22}$

La réflexion se développant toujours dans le cadre de la représentation phonologique, c'est-à-dire de la formalisation d'opérations linguistiques ou cognitives qui doivent rendre compte de la capacité de langage du locuteur, les autosegments sont des objets abstraits. Ils résultent d'une opération d'analyse qui isole un aspect abstrait au sein du continuum phonique (l'accent par exemple, ou encore le ton). La qualification d'autosegment résulte du postulat d'autonomie relative de l'objet isolé par rapport aux autres éléments du continuum phonique (les tons sont considérés comme des éléments distincts de la voyelle qu'ils affectent et, à ce titre, doivent être isolés).

Pour marquer graphiquement l'autonomie des types d'objets identifiés, on les place sur des lignes différentes, dites lignes ou suites autosegmentales, telles la ligne des tons, la ligne des accents, la ligne des segments (vocaliques ou consonantiques) ou encore les lignes créées pour supporter différents types d'objets identifiés: autosegment syllabique, nasalité, aspiration etc. (voir schéma présenté ci-dessous). Chaque ligne regroupe des éléments qui ont des «propriétés fonctionnellement identiques» ${ }^{23}$.

De ce point de vue, le modèle S.P.E. est considéré comme une suite phonologique unilinéaire, et non autosegmentale, puisque les unités phonologiques sont des matrices réunissant à la fois des traits suprasegmentaux («accentué», «syllabique») et des traits segmentaux (traits phoniques binaires).

Reste bien sûr à garantir la cohérence entre ces différentes lignes autosegmentales. $C^{\prime}$ est le rôle d'une ligne centrale, définie comme la «représentation temporelle de l'unité phonologique considérée» ${ }^{24}$, qui sera assimilée, selon les auteurs et la période, à la ligne des phonèmes (autosegments) ou bien à une succession de positions consonantiques et vocaliques (Clements et Keyser ${ }^{25}$, MacCarthy ${ }^{26}$ ) puis, quand le rôle de la structuration syllabique sera reconnu et pris en compte en phonologie générative,

${ }^{21}$ GOLDSMITH, John, Autosegmental Phonology, PhD thesis, MIT, Cambridge Ma., 1976.

22 On délaissera ici les débats menés entre tenants de la théorie autosegmentale et tenants de la «compositionnalité «(ou «constituance») qui touchent aussi bien la phonologie que la syntaxe générative, voire les thèses de la recherche en cognition, et dont la portée échappe pour l'heure à l'auteur de ces lignes.

${ }^{23}$ LAKS, Architecture..., p. 9.

${ }^{24}$ LAKS, Architecture..., p. 10.

25 CLEMENTS, George et S.J KEYSER., CV Phonology. A Generative Theory of the Syllabe, MIT Press, Cambridge Mass., 1982

${ }^{26}$ McCARTHY, John, 1979, Formal Problems in Semitic Phonology and Morphology, PhD thesis, MIT 
à une ligne de «positions pures» (Kaye, Lowenstamm et Vergnaud $\left.{ }^{27}\right)$. Cette ligne des positions (marquées par de simples points ou des $x$ dans les schémas) qui recevra le nom de «squelette» est un ancrage autour duquel vont s'articuler les différentes lignes autosegmentales définies par le phonologue (chaque couple squelette-ligne constituant un «plan»), un peu à la manière de la reliure d'un livre qui tient ensemble les pages qui le composent.

La représentation est dite dès lors pluri- ou multilinéaire et, pour les cas complexes, tridimensionnelle, quand elle affecte l'allure générale d'un prisme dont l'un des côtés représente la ligne des positions, les autres étant les lignes autosegmentales, chaque position du squelette étant reliée par une portion de droite à l'autosegment, ou aux autosegments, qui l'occupe(nt).

Le schéma ci-dessous illustre la représentation autosegmentale du mot hébreu kittam $^{28}$ :

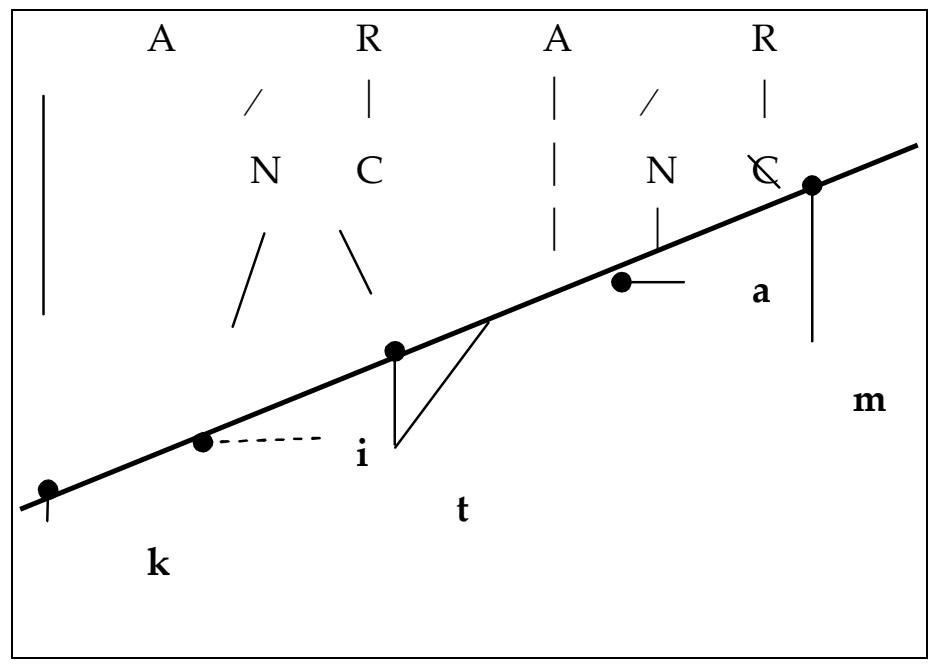

Les «positions» du squelette sont marqués par des points et correspondent aux «nœuds terminaux» des arbres syllabiques (Attaque et Rime se divisant en Noyau et Coda); à chaque position est rattaché un segment phonème (un plan pour les consonnes, un pour les voyelles).

Dans ce type de schéma, une «position» peut être simultanément concernée par des segments de nature différente: voyelles, tons, accent, structure syllabique, rythme, prosodie etc., la liste étant à la discrétion de l'analyste. Inversement, une unité phonique peut être rattachée à plus d'une position: c'est la situation postulée par la théorie pour les voyelles longues par exemple, qui sont rattachées à deux positions du squelette et occupent deux places dans la représentation temporelle, contre une seule pour les voyelles brèves, ou encore pour les consonnes géminées comme c'est le cas ici pour /t/ qui occupe deux positions. Selon certaines extensions du modèle, des objets distincts figurant sur une même ligne autosegmentale peuvent être rattachés à une position unique du squelette (les affriquées sont ainsi des segments distincts rattachés à une seule position). Enfin, une unité phonique peut être sans attache fixe: c'est le cas

27 KAYE, Jonathan, Jean LOWENSTAMM et Jean-Roger VERGNAUD, 1990, Constituent structure and government in phonology, Phonology 7(2), p. 193-231

28 Source : Pierre ENCREVÉ, La liaison avec et sans enchainement: phonologie tridimensionnelle et usages du français, Paris, Ed. du Seuil, 1988, 317 p. 


\section{MANUSCRIT AUTEUR}

des fameuses «consonnes flottantes» qui seraient en jeu dans les phénomènes de liaison $\mathrm{du}$ français... Cette conception autosegmentale va produire notamment le Principe de Contour Obligatoire selon lequel deux positions consécutives quelconques doivent obligatoirement «faire contour» (ne pas être occupées par deux éléments phonologiques identiques).

Traitant de ce sujet, Pierre Encrevé précise que «le squelette de positions pures correspond au nombre de positions potentielles définissant un mot donné pour un locuteur donné (car il ne va pas de soi que tous les locuteurs partagent des représentations lexicales absolument identiques): nous entendons par là le nombre d'unités possible que le locuteur attribue intuitivement à un mot mémorisé» ${ }^{29}$, et comme l'existence même de cette représentation lexicale intuitive et sa nature sont problématiques, il prend soin de préciser que «parce que rien n'est plus abstrait qu'un squelette de positions pures en phonologie, le risque est (...) grand de tomber dans l'arbitraire» ${ }^{30}$, ce dont on conviendra volontiers, d'autant plus, précise-t-il, que «l'habitude a été si bien prise en phonologie générative de poser des segments abstraits, qu'un objet encore plus abstrait pourrait ne pas susciter de questions particulières quant à sa réalité» 31 ... Ces remarques n'entament cependant pas son enthousiasme puisqu'il écrit à quelques pages de là: «En séparant les unes des autres, la représentation d'une suite de positions abstraites absolument distinctes des unités segmentales, syllabiques ou autres, les interprétant, chacune dans son ordre, fait faire à la phonologie un progrès révolutionnaire dont la portée ne devrait pas être limitée au cadre théorique dans lequel cette notion d'un squelette de positions pures de tout contenu phonétique a été pensée, mais devrait s'imposer - dans son principe si ce n'est dans sa formalisation actuelle - à toute théorie phonologique» ${ }^{32}$.

Force est de constater que ce "progrès révolutionnaire» ne s'est pas répandu jusqu'à présent en dehors du cadre générativiste, ce qui n'est pas rien toutefois étant donné le rapport des forces entre écoles linguistiques dans le monde... Et au sein de ce seul courant, l'accord sur ce qui constitue un squelette est loin d'être acquis: la bibliographie abonde en analyses discordantes quant au nombre de positions qu'il faut attribuer à tel ou tel mot - selon l'interprétation qui est faite à chaque fois de «l'intuition» du locuteur.

\section{Le segment ou le phonème?}

Une autre difficulté majeure du modèle S.P.E. résidait bien entendu dans son refus obstiné du phonème. Différents auteurs ${ }^{33}$ se sont penchés sur les motifs d'un tel rejet chez Chomsky et Halle, mais l'histoire de cette épuration radicale de la phonologie reste à écrire ${ }^{34}$. A l'issue d'un cycle de réflexion, Chomsky et d'autres générativistes en

\footnotetext{
${ }^{29}$ ENCREVÉ, La liaison..., p. 153.

30 ENCREVÉ, La liaison..., p. 151

31 ENCREVÉ, La liaison..., p. 153

32 ENCREVÉ, La liaison..., p. 146

${ }^{33}$ Claude Hagège a bien résumé l'enjeu de ce refus de la phonologie en y voyant «une de ses croisades les plus obstinées et les moins décisives» de la grammaire générative (La grammaire générative : réflexions critiques, Paris, P.U.F., coll. «Le linguiste», p. 171-172).

${ }^{34}$ Voir les quelques éléments historiques que donne Pierre ENCREVÉ dans son article L'ancien et le nouveau..., Langages $n^{\circ} 125$, p. 101.
} 
viennent à réintroduire le phonème dans leur terminologie, comme réalité empirique et comme notion qui peut être déduite de l'application des règles de la composante phonétique (le phonème serait alors le résultat de l'application de ces règles), sans toutefois reconnaître un niveau particulier pour le phonème. Le phonème sans la phonologie en quelque sorte.

Les évolutions des vingt dernières années semblent suivre deux tendances. La première vise à préserver la notion chomskyenne de «segment» en lui donnant la consistance substantielle que le modèle S.P.E. lui refusait; différentes propositions ont été faites dans ce domaine, qui s'organisent grossièrement autour d'un pôle «traits et règles» et d'un autre «principes et paramètres». L'autre tendance concerne les travaux qui veulent réintroduire le phonème dans la théorie en tant que catégorie cognitive.

\section{De quoi sont faits les segments?}

Jusqu'à ce jour la plupart des auteurs générativistes anglo-saxons semblent maintenir le cap d'une phonologie sans phonème (une théorie des traits), essentiellement parce que leur analyse s'inscrit dans un programme que Clements énonce dans ces termes: «Une théorie des traits phonologiques (...) devrait être capable, entre autres, de distinguer: a) tous les segments consonantiques et vocaliques qui sont phonémiquement contrastifs dans les langues du monde; b) tous les groupes de sons qui fonctionnent ensemble dans les règles phonologiques (les classes naturelles de sons); c) tous les traits qui fonctionnent comme une unité dans les règles phonologiques.» ${ }^{35}$ Dans une telle perspective, qui travaille à l'aide de règles et de traits universels (hiérarchisés et non abstraits), il est cohérent d'ignorer la notion de phonème, dont la définition structurale renvoie nécessairement $\mathrm{au}(\mathrm{x})$ système(s) propre(s) à chaque langue.

Clements propose de hiérarchiser les traits phoniques et de les répartir en différentes «classes majeures», sur plusieurs lignes (tiers, parfois traduit par tires) entendues comme des niveaux d'analyse distincts: on parlera ainsi de niveaux laryngal, supralaryngal, de mode d'articulation («manner tier») et de lieu d'articulation («place tier»), manière comme une autre de réintroduire certaines solidarités structurelles que l'analyse en traits binaires tentait de nier; ses propositions tendent aussi à faire usage de traits non abstraits, c'est-à-dire définis par des propriétés acoustiques et articulatoires (réputées valables pour toutes les langues).

Les tenants de cette position évoquent en sa faveur le constat que les changements phonétiques historiques, les processus phonologiques, mettent en jeu d'une part certains traits phoniques (et non l'ensemble des traits définissant le phonème pragois), donc un niveau «infraphonémique», et d'autre part des ensembles de sons, les «classes naturelles». De quelque côté qu'on aborde une telle proposition, on voit difficilement en quoi elle peut constituer une nouveauté par rapport aux thèses structurales et notamment fonctionnalistes: les changements phonétiques affectant les phonèmes se

\footnotetext{
35 Voir la synthèse donnée dans l'article Lieu d'articulation des consonnes et des voyelles: une théorie unifiée, dans Bernard LAKS, Architecture des représentations phonologiques, p. 101-145. Dans cet article Clements annonce une proposition qui selon lui permet de rendre compte de la différence entre consonnes et voyelles; différence qui semble se résumer finalement à la distinction entre 'trait de lieu d'articulation des consonnes' et 'trait de lieu d'articulation des voyelles': dire qu'on reste sur sa faim est un euphémisme...
} 
manifestent bien par des modifications de traits, la phonologie historique le démontre amplement, et ils concernent généralement des ensembles de phonèmes partageant les mêmes traits (qu'on relise Martinet dans l'Economie des changements phonétiques).

La référence aux «processus phonologiques» (dynamiques) plutôt qu'aux systèmes (statiques) est présentée comme une «réorientation fondamentale de la phonologie» ${ }^{36}$, qui serait à l'origine notamment de la contestation par Chomsky de l'existence du niveau phonémique.

S'il est utile de faire de l'histoire, autant la faire de manière non téléologique (la phonologie générative comme aboutissement logique et sans rupture de l'histoire de la phonologie du $20^{\text {e }}$ siècle et des thèses saussuriennes... $)^{37}$ et essayer de savoir si le prétendu statisme n'est pas tant une caractéristique du système phonologique pragois qu'un effet de la conception étroite qu'en eût une partie des linguistes américains, auxquels Chomsky prétendait répondre, en prenant pour argent comptant leur interprétation restrictive.

\section{Les positions «unaristes»}

D'autres auteurs, critiquant le caractère arbitraire des règles et des traits phonologiques généralement retenus en phonologie générative, tentent de rendre compte des particularités de chaque langue dans un cadre qui concilie les ambitions universalistes et la variété des phénomènes observés. Schane ${ }^{38}$ puis Kaye, Lowenstamm et Vergnaud ${ }^{39}$ ou encore van der Hulst ${ }^{40}$ ou Carvalho ${ }^{41}$ mènent une critique radicale de la notation binariste et consacrent son abandon au profit d'une «approche unariste» qui, pour qualifier les voyelles par exemple, n'utilise que trois «éléments» I, A, U «dont le contenu phonologique respectif peut se traduire par «[avant]», «[bas]» et «[arrondi]».»42 Carvalho, admettant comme constat de départ que «les langues varient quant à leur structure phonologique», propose une interprétation des représentations phonologiques (vocaliques en l'occurrence) dans le cadre dit

36 LAKS, Bernard, Un siècle de phonologie: quelques questions permanentes, Modèles linguistiques, Paris, 2000, p. 93.

37 Voir dans cette veine l'article de Bernard LAKS, Un siècle de phonologie..., et celui de Pierre ENCREVÉ, L'ancien et le nouveau..., Langages n 125 , p. 100-123. Ces auteurs insistent à juste titre sur le fait que certaines préoccupations de la phonologie sont remarquablement stables dans le temps et à travers les écoles, et ils rappellent utilement que la prétendue innovation (rupture ?) chomskyenne en phonologie doit être pesée en fonction d'arguments historiques et théoriques sérieux. On ne peut qu'être d'accord avec la démarche; on est moins convaincu en revanche quand Laks tente de démontrer que la proposition de Chomsky «s'inscrit parfaitement dans la logique du programme phonologique inauguré au début du siècle», une quasi filiation unissant ses positions à celles de Saussure, ce dernier se voyant attribuer a posteriori un point de vue «pratiquement autosegmental» sur la phonologie...

38 SHANE, S. A., The fundamentals of particle phonology, Phonology yearbook 1, 1984, p. 129-155.

${ }^{39}$ KAYE, Jonathan, Jean LOWENSTAMM et Jean-Roger VERGNAUD, The internal structure of phonological elements: a theory of charm and government, Phonology yearbook 2, p. 305-328.

40 Van der HULST, Harry, Les atomes de la structure segmentale: composants, gestes et dépendance, dans LAKS \& RIALLAND, Architectures des représentations phonologiques, p. 255290.

41 BRANDÃO de CARVALHO, Joachim, Primitives et naturalité, Langages n 125, p. 14-34.

42 CARVALHO, Primitives..., p. 17. 
«principes et paramètres» ${ }^{43}$. Le "principiel» concerne la définition des éléments avec lesquels travaille la phonologie; les particularités des différentes langues découlent de «paramètrisations» différentes. Selon le «modèle IAU» complété, une voyelle est une sorte de matrice de primitives (dont l'existence et le nombre sont postulés), du type «particule», «poids», «activité», «gouvernement». À titre d'exemple et en simplifiant fortement, trois particules seulement - deux pour la «couleur» (I U) et A pour la «sonorité» 44 - auxquelles s'ajoute une particule vide $\mathrm{v}^{0}$, entrent dans différentes combinaisons (à raison de trois occurrences maximum) pour produire toutes les voyelles: /i/ égale $\mathrm{I}+\mathrm{I}+\mathrm{I}, / \mathrm{o} /$ égale $\mathrm{A}+\mathrm{U}+\mathrm{U}$, etc. Dans une certaine mesure, les consonnes peuvent également être décrites par un tel appareillage conceptuel. Les paramètres invoqués pour rendre compte des différences entre les systèmes phonologiques des langues mettent en jeu des combinaisons particulières de «propriétés» des éléments que sont l'activité et le poids.

Portons au crédit de chacune des approches présentées ci-dessus le souci de définir des structures qui peuvent recevoir des interprétations phonétiques «naturelles». La lecture attentive des articles cités montre une tendance à donner aux «segments» manipulés une forme d'existence systémique qui les rapprocherait du phonème structuraliste. On trouve ainsi sous la plume d'Anderson et Durand une description du système vocalique du yawelmani (où la composition des voyelles est exprimée dans les termes du «modèle IUA»). ${ }^{45}$

Les voyelles du yawelmani selon Anderson et Durand

$$
\{\mathrm{i}\} / \mathrm{i} / \quad\{\mathrm{u}\} / \mathrm{u} /
$$

$$
\{\mathrm{u}, \mathrm{a}\} / \mathrm{o} /
$$

$\{a\} / a /$

Le commentaire accompagnant le schéma ci-contre précise que /i/ (composé de l'élément \{i\}) est isolé alors que /u/ et /a/ sont reliés par /o/ qui combine les composants $\{u, a\}$; que le trait $\{i\}$ est redondant; que la représentation de /i/ pourrait être vide \{\} car de toute façon différente des autres voyelles du yawelmani... Par un biais fort détourné, on retrouve ici un principe essentiel de toute la phonologie structuraliste, à savoir qu'un phonème est ce que les autres ne sont pas et que son degré d'intégration dépend du réseau d'oppositions dans lequel il s'insère...

43 BRANDÃO de CARVALHO, Joachim, De quoi sont faites les voyelles? dans LAKS et PLENAT (dir.), De natura sonorum, p. 65-100

${ }^{44}$ Chez van der HULST deux éléments seulement se combinent pour former des voyelles, par ex. : $[\mathrm{e}]=\mathrm{I}+\mathrm{A},[\boldsymbol{x}]=\mathrm{A}+\mathrm{I}$ (l'ordre a son importance car il indique l'élément «gouverneur» et l'élément «dépendant»).

45 ANDERSON, John et Jacques DURAND, Segments non spécifiés et sous spécifiés en phonologie de dépendance, dans LAKS et RIALLAND, Architectures..., p. 233-253. 


\section{Le phonème, réalité cognitive}

Certaines évolutions récentes de la phonologie générative semblent montrer une attitude plus prudente et en apparence conciliatrice envers le phonème. Des auteurs comme Laks ${ }^{46}$ font une référence explicite à la phonologie structurale, et même à André Martinet, quand il s'agit de faire l'inventaire des phonèmes d'une langue, et la distinction entre réalité fonctionnelle du phonème et variété de ses manifestations phoniques est clairement établie. Le phonème est réintroduit en tant que marque d'une activité cognitive: un mécanisme de «filtrage cognitif» interviendrait pour ne retenir dans la variété des réalisations sonores que ce qui est pertinent dans une langue donnée. Dans cette perspective, qui redéfinit la phonologie comme une science de la cognition et du fonctionnement mental, le phonème doit être appréhendé du point de vue des représentations linguistiques qui sous-tendent l'activité langagière. Laks y voit une continuité logique avec la conception saussurienne des systèmes sonores (linguistiques) comme «systèmes abstraits de relations n'existant que dans les cerveaux des locuteurs» ${ }^{47}$. Pour les tenants inconditionnels de l'innéité, l'existence du phonème relève de l'ordre du "principe», c'est-à-dire des caractéristiques de la capacité universelle de langage: chaque humain ayant à la naissance la capacité de se représenter plusieurs centaines de phonèmes, capacité ramenée à l'emploi de quelques dizaines d'éléments seulement par l'apprentissage de la (ou des) langue(s) première(s), selon les "paramètres» particuliers de chaque langue. Les phonèmes sont conçus comme des entités abstraites, essentiellement mentales, des classes d'équivalence reconstruites à partir de sons physiquement différents, sans qu'on sache réellement ce qui fonde de tels regroupements. Or c'est justement l'apport de la phonologie (pragoise) que d'avoir montré que ces «classes d'équivalence» se constituent dans l'activité langagière elle-même à travers les oppositions d'unités significatives qu'elles permettent, ce qui est stricto sensu définir les phonèmes comme n'étant «que ce qu'ils font». De la proposition saussurienne, qui insiste fondamentalement sur les notions de système et d'opposition, l'orientation cognitiviste ne semble retenir que son inscription «dans les cerveaux des locuteurs». Ce qui est évacué, ou plutôt ramené au statut de paramètre, c'est le caractère social, et dynamique, du phonème tel que le conçoit la phonologie pragoise et sa définition comme unité oppositive au sein d'un système, ensemble d'habitudes articulatoires propres à une langue. En retrouvant sa définition mentaliste des débuts de la phonologie (peu importe qu'on le qualifie maintenant de réalité cognitive) le phonème est ramené au statut d'hypothèse parmi les autres dans le vaste champ de réflexion sur les représentations linguistiques.

\section{La syllabe}

Autre grande absente de la phonologie générative première époque, la syllabe est devenue, paradoxalement, une notion pivot de la phonologie générative contemporaine. La réflexion générativiste sur le sujet s'est développée à partir de la

46 LAKS, Bernard, Phonologie et cognition, dans Yves MICHAUX (éd.) Université de tous les savoirs: Qu'est-ce que l'humain ? vol. 2, Paris, Editions Odile Jacob, 2000, p. 69-84.

47 LAKS, Bernard, Un siècle de phonologie: quelques questions permanentes, Modèles linguistiques, Paris, 2000, p. 75-102. 
critique du traitement des phénomènes accentuels de l'anglais proposé par le modèle S.P.E.

Dans le modèle initial la syllabe est ignorée et elle sera intégrée tardivement par Chomsky et Halle comme une caractéristique du segment vocalique: la syllabicité. Les travaux des tenants de la "phonologie générative naturelle» comme Vennemann ou Hooper, puis ceux de Goldsmith, de Liberman et Prince ou encore de Kahn vont imposer progressivement une nouvelle organisation de la phonologie générative autour de la syllabe, en y intégrant la prise en compte de la «hiérarchie de sonorité». La syllabe est analysée en deux constituants ou nœuds, le premier composé d'une attaque et d'une rime, le deuxième d'un noyau obligatoire et d'une coda facultative. Bien qu'appuyé sans grande discussion sur des arguments phonétiques, la syllabe dont il est question est une syllabe «théorique», une syllabe «type» différente de «la syllabe phonétique telle qu'on la rencontre dans les langues» comme l'écrivait Grammont ${ }^{48}$.

On connaît la prudence des structuralistes et des phonéticiens qui se sont penchés sur le sujet, et parmi d'autres Martinet, Malmberg, Ladefoged ou Jacqueline Thomas: si la réalité empirique et opérationnelle de l'objet syllabe est reconnue, le scepticisme l'emporte largement quant à la possibilité de définir les faits (physiques, phonétiques) qui réalisent cette «unité abstraite et fonctionnelle» (Malmberg). La phonologie structurale utilise la syllabe comme un objet théorique d'un genre un peu particulier, utile, mais dont la définition reste problématique. En tout cas, son statut est rediscuté langue par langue, en fonction des systèmes phonologiques rencontrés et des latitudes distributionnelles attestées.

Rien de tel chez les générativistes qui postulent parmi les caractères universels du langage une structure syllabique minimale, conçue comme un modèle sous-jacent, obligatoirement représentée par une attaque et une rime et produisant un schème canonique $\mathrm{CVC}, \mathrm{CV}(\mathrm{C})$ ou strictement de type $\mathrm{CV}$ selon les auteurs et les époques. C'est sur cette base que se sont développés aux cours des deux décennies passées les travaux générativistes concernant l'analyse des structures syllabiques de différentes langues (un vaste effort pour ramener les exceptions, forcément nombreuses, au modèle sous-jacent postulé), des phénomènes à cheval sur la diachronie et la synchronie comme l'allongement compensatoire, des phénomènes de diphtongaison, etc.

Intégrée à la théorie autosegmentale, la suite syllabique y occupera un temps une place importante, pouvant servir, on l'a vu, de ligne de positions pour certains auteurs.

Le postulat de la syllabe minimale a eu une importance particulière dans la réflexion sur la notion de squelette; en effet comme l'explique Angoujard ${ }^{49}$, pour définir ce qu'est une structure rythmique au sein d'un mot, on s'est appuyé sur l'hypothèse d'alternances régulières de positions fortes et faibles, elles mêmes assimilées à la position des voyelles et des consonnes, les consonnes occupant des positions faibles et les voyelles des positions fortes. La syllabe minimale étant CV on en déduit donc que toute séquence rythmique commence par une position faible (attaque). Angoujard tient tout de même à apporter la précision suivante: "[Le modèle rythmique] n'impose absolument pas qu'un mot commence par une consonne (ce qui serait à l'évidence,

48 GRAMMONT, Maurice, Traité de phonétique, Paris, Delagrave, 1933

${ }^{49}$ ANGOUJARD, Théorie de la syllabe 
empiriquement faux) mais qu'une séquence rythmique commence par un creux.» ${ }^{50}$. D'où il découle qu'un mot à voyelle initiale présente une «attaque vide», position qui fonde tous les travaux générativistes sur les phénomènes de jointures dans la chaîne par exemple (voir notamment les travaux d'Encrevé sur la liaison en français). Cette hypothèse a un statut fort mal assuré et comme le fait remarquer Carvalho: «les théories optimalistes de la syllabation énoncent, par exemple, que la syllabe (optimale) doit avoir une attaque; elles n'expliquent pas pourquoi et la contrainte est dès lors arbitraire. Une proposition alternative consisterait précisément à se demander si les effets de telles contraintes peuvent être dérivés d'une théorie des primitives» ${ }^{51}$.

La réflexion sur la syllabe est loin d'être achevée, puisque comme l'écrit Angoujard, il est probable que cet «objet n'existe pas, qu'il est au mieux, que l'effet variable des interactions entre plusieurs objets élémentaires» ${ }^{52}$. Pour lui, il s'agit de la grille rythmique, du segment (conçus comme le résultat de la composition d'éléments) et de la courbe prosodique; pour Klein c'est une «interface de la production et de la réception phoniques ${ }^{53}$... On ne peut qu'être frappé par le contraste entre l'affirmation péremptoire de l'universalité de l'objet et la difficulté persistante à le saisir et à se mettre d'accord sur sa définition au sein d'une même théorie. Et dans le cadre d'une «théorie des primitives» ou dans un autre on est en effet demandeur de quelques arguments en faveur de telles conceptions.

\section{Conclusion}

Quels enseignements tirer d'un survol aussi rapide et partial d'un domaine immense, où la variété et les divergences l'emportent bien souvent sur les points d'accord clairement identifiés entre les tenants de ces prétendues «nouvelles phonologies»?

D'un certain point de vue, les évolutions de la phonologie (post?) générative pourraient être lues comme une convergence possible avec les thèses structuralistes, mais les chemins empruntés sont tellement sinueux et le rapprochement si ténu qu'on peut encore aujourd'hui se demander s'il y aurait quelque bénéfice à confronter sérieusement les positions fonctionnalistes à ce courant.

L'hypothèse d'une «phonologie universelle» est et restera un désaccord fondamental, et l'orientation cognitiviste - qui n'est partagée qu'à des degrés variables par les générativistes eux-mêmes, semble poser plus de problèmes qu'elle n'en résout (ne serait-ce qu'en délaissant le terrain relativement ferme - pour un fonctionnaliste du matériel sonore pour celui encore bien flou - pour tout le monde cette fois - des opérations mentales).

\footnotetext{
50 ANGOUJARD, Théorie de la syllabe, p. 82.

${ }^{51}$ CARVALHO, Langages n ${ }^{\circ} 125$, p. 32.

52 ANGOUJARD, Théorie de la syllabe, p. 24.

53 KLEIN Marc, La syllabe comme interface de la production et de la réception phoniques, in LAKS, De natura sonorum, p. 101-141.
} 
La phonologie fonctionnelle, et plus largement la linguistique fonctionnelle, ne se donne pas pour but de rendre compte «exhaustivement et explicitement de la compétence du sujet parlant ${ }^{54}$.

Une hypothèse comme celle $\mathrm{du}$ «squelette de positions pures» constitue évidemment un autre point de divergence, et pour diverses raisons.

- Parce qu'elle repose sur l'hypothèse cognitiviste d'une «représentation intuitive» $\mathrm{du}$ locuteur qui reste étrangère aux stipulations de la linguistique fonctionnelle.

- Parce qu'elle pose comme acquis que le mot (phonologique ou non) est le format de cette représentation.

- Parce qu'elle entre en contradiction avec une conception dynamique et collective des faits linguistiques : face à des évolutions diachroniques du type /mestr/ > /me:tr/ > /metr/ «maître», cette dernière forme n'étant plus distincte aujourd'hui dans les usages majoritaires du français de /metr/ «mètre», «mettre»; quelles peuvent être l'utilité et la réalité de l'hypothèse d'une «représentation intuitive» du type /me(s)tr/ qui demeurerait inchangée dans la tête des locuteurs en dépit des réalités systémiques observables (absence d'opposition de longueur vocalique en français contemporain) ?

- Parce qu'elle découle d'un refus des notions de signe et de variante du signifiant: si l'on admet que /pəti/ et /pətit/ sont des variantes formelles d'un même signifié «petit», il n'est aucun besoin de postuler une «consonne flottante»/t/ et des «attaques vides» à combler dans les mots à voyelle initiale pour rendre compte des cas de «liaison» en français par exemple.

Et contrairement à ce que veulent croire certains générativistes, noter ces variantes d'un signifiant et leurs conditions d'apparition, ce n'est pas de la part des structuralistes se limiter à une "petite portion de l'objet à décrire» ${ }^{55}$. Selon des critères de pertinence clairement définis (la pertinence communicative), montrer en quoi les structures internes des langues sont des réponses, provisoires et parfois instables, aux besoins communicatifs va au-delà de la description: le linguiste accède de cette manière aux facteurs explicatifs de l'organisation et de la transformation des langues, aussi bien en synchronie qu'en diachronie.

Il s'agit bien d'un face à face entre deux conceptions radicalement distinctes de l'activité langagière: d'un côté, chez les fonctionnalistes, un primat accordé à la régulation sociale du comportement linguistique (l'acquisition - stabilisée ou non, mais socialement contrôlée - des signes linguistiques propres à telle langue et des variantes formelles d'un même signifié dans leurs conditions d'emploi); de l'autre un modèle génératif voulant rendre compte des variations phoniques à partir d'une suite fixe de morphèmes (le «gabarit» du mot).

La divergence se manifeste également quant au rapport entre ce qui relève de la chaîne sonore et ce qui concerne l'économie phonique générale de la langue. Pour la phonologie fonctionnelle, la stabilité des formes phoniques tout comme leurs

54 Carole Paradis note fort justement qu'il s'agit d'une différence fondamentale entre phonologie générative et phonologie fonctionnelle, et que cela explique que le point de vue fonctionnaliste ignore les «règles» censées formaliser les liens entre les «représentations» que sont les phonèmes, les lexèmes, les monèmes, etc. Voir Carole PARADIS, Phonologie générative multilinéaire, dans Jean-Luc NESPOULOS (éd.), Tendances actuelles en linguistique générale, Delachaux et Niestlé, p. 11-45.

${ }^{55}$ F. Dell, cité par Angoujard dans La phonologie déclarative, Langages n ${ }^{125}$, p. 40 
transformations dans le temps résultent des sollicitations contradictoires de la «tendance au moindre effort» et de la nécessité de maintenir les distinctions phoniques utiles à la communication; la phonologie générative y voit un résultat de règles mécaniques qui touchent la forme sans affecter le schème sous-jacent.

$\mathrm{Au}$ vu de telles divergences, on pourrait tirer la conclusion que rien ne peut rapprocher ces deux types de phonologie; cependant, certaines évolutions méritent d'être signalées: la notion de «phonème» n'est sans doute plus aussi éloignée aujourd'hui des positions de la phonologie générative que dans les années triomphantes du «Hallo-Chomskysme»; l'abandon du binarisme semble s'affirmer ; l'idée d'une phonologie inféodée à la syntaxe n'est plus réellement assumée ; certains auteurs rejettent explicitement l'analyse en deux niveaux (sous-jacent et surface)... ${ }^{\mathbf{5 6}}$

Par ailleurs, l'hypothèse d'une structuration de ce qui se présente comme linéaire, la chaîne parlée, si elle n'est pas nouvelle, est en elle-même séduisante et, au-delà de l'arbitraire et du conventionnel, il y a sans doute une réflexion intéressante à mener sur ce qui rend possible certaines combinaisons de phonèmes dans une langue et pas dans une autre, sur ce qui provoque l'harmonie vocalique dans certaines langues et l'empêche ailleurs, sur les habitudes de la chaîne qui favorisent tel ou tel type d'allongement compensatoire, ou encore sur ce qui fait la pulsation rythmique particulière de telle ou telle langue... Dans ces domaines, les phonologies post génératives ont ouvert quelques pistes qui méritent examen.

Le projet théorique générativiste est un épisode important de l'histoire récente de la linguistique, sinon pour la connaissance de ce que sont les langues, du moins pour la réflexion sur la manière dont se forment, évoluent et se propagent les idées en sciences. Il y a vingt-cinq ans Georges Mounin ${ }^{57}$ notait qu'il était bien difficile de savoir si les thèses de Chomsky avaient produit $d u$ nouveau en linguistique - du réellement nouveau et pas seulement déclaré tel par les tenants de la théorie. Aujourd'hui le bilan générativiste orthodoxe - inscrit dans la lignée directe de Chomsky et Halle - paraît bien maigre en matière linguistique, là où il nous est possible de juger ${ }^{58}$; les travaux issus des «nouvelles phonologies» - qui ne le sont que pour ceux qui ont du faire l'effort de rompre avec la tradition S.P.E. - méritent notre attention car des linguistes qui sont engagés dans cette voie sont confrontés à des problèmes qui, pour une part, sont des problèmes communs à tous les phonologues. La contribution de la phonologie générative à la recherche en cognition devra être évaluée également, avec d'autres compétences que les nôtres.

Si de tels problèmes peuvent intéresser la linguistique fonctionnelle, en tout état de cause il s'agit surtout de relancer la discussion entre fonctionnalistes sur des problèmes de phonologie, un tel débat pouvant inclure un volet consacré à l'évaluation d'autres

56 Voir la prise de position d'Angoujard dans son article, La phonologie déclarative, Langages $\mathrm{n}^{\circ}$ 125 , où il écrit «il ne peut exister qu'un seul niveau de représentation et ce dernier ne saurait être distinct des structures de surface».

57 MOUNIN, Georges, La linguistique au XXe siècle, Paris, P.U.F., coll. «Le linguiste», 1975, 253 p.

58 On se rappelle les mots de Robert A. HALL pour qui, dans The sound pattern of English, Chomsky et Halle réussissaient à prouver deux choses seulement à propos de l'anglais: 1) que la place de l'accent tonique est phonologique et 2) que les alternances morphologiques actuelles reflètent des segmentations phonémiques remontant à l'ancien anglais, «Mais [précisait Hall] on savait tout cela depuis longtemps»... (voir Robert HALL, 'Underlying representation' and observable fact in phonology, Journal of English Linguistics, 7, 1973, p. 21-42). 
propositions théoriques. Alors, comme nos devanciers structuralistes l'ont fait, il faut continuer de lire les générativistes, sereinement et sérieusement. 